\title{
El Botón Rojo de RTVE: una experiencia de implantación de HbbTV en España
}

\section{RTVE's Red Button: a HbbTV implementation experience in Spain}

\author{
David Varona Aramburu. Universidad Francisco de Vitoria
}

Recibido: 2-I-2014 - Aceptado: 28-V-2014

Resumen:

El ecosistema televisivo se ha visto alterado con la aparición de las televisiones conectadas, aparatos capaces de conectarse a Internet a través de diferentes sistemas. Uno de ellos es el HbbTV, Hybrid Broadcast Broadband Television. Impulsado sobre todo por la UE y la industria, supone un cambio importante en la forma de consumir televisión y abre la red a públicos nuevos. Esta investigación estudia la implantación de HbbTV llevada a cabo en RTVE. El trabajo propone la descripción del servicio denominado Botón Rojo de RTVE y el análisis de la respuesta de la audiencia en sus primeros meses de vida. La relevancia del estudio estriba en el papel de locomotora del sector audiovisual que se atribuye frecuentemente a TVE.

Palabras clave:

HbbTV, televisión conectada, botón rojo, convergencia, televisión interactiva.

\section{Abstract:}

The television ecosystem has been altered by the emergence of so-called connected televisions or smartTVs, devices capable of connecting to the Internet in different ways. One of these is the HbbTV, Hybrid Broadcast Broadband Television. Backed by the EU, it constitutes a relevant change in the way people consume television and a way for new audiences to reach the Web. This research focuses on the HbbTV implementation held by Radio Televisión Española (RTVE) in 2013. The paper includes a formal description of the Botón Rojo (Red Button) service and an evaluation of viewers" reactions and feedback to this new feature. Its significance lies in the central role of RTVE, a well-known powerhouse for research and development, in the Spanish audiovisual market.

Key words:

Smart tv, red button, interactive television, convergence, interactive television. 


\section{Introducción y estado de la cuestión}

La idea de acceder a Internet desde la televisión ha cobrado forma durante el último lustro, pero no es para nada nueva. Ya Nicolás Negroponte (2000) hablaba de la televisión como un ordenador y señalaba que "la clave del futuro de la televisión es dejar de pensar en ella como tal y concebirla en términos de bits”. Desde entonces, el proceso de convergencia entre ordenadores y televisiones, y, sobre todo, entre Internet y la televisión, no ha hecho más que acelerarse y hoy en día es difícil deslindar dónde empieza Internet y dónde la televisión, toda vez que los productos televisivos circulan por la red, animados por la explosión de las redes sociales y la telefonía móvil. Y, más allá: Internet empieza a colonizar las de televisiones, un territorio que hasta ahora parecía fuera de su alcance.

Se avanza ahora en un modelo de "hipertelevisión" (Scolari, 2008). En un mercado digital poblado por canales IPTV, agregadores de contenidos, vídeo on demand, over the top, y otras soluciones ubicuas y baratas, es necesario estar de acuerdo con José María Álvarez Monzoncillo (2011), que señala que "todo es televisión, pero poco se parece a la vieja televisión analógica”. Este autor apunta que estos “new media” suponen un cambio profundo y "alteran las formas de recepción, el comportamiento de las audiencias, los negocios de los productores, programadores y difusores”.

Es interesante el planteamiento de Álvarez Monzoncillo, pues toca uno de los elementos clave de la ecuación: la audiencia. Los espectadores se han acostumbrado con facilidad al esquema de interactuación propio de Internet y exigen lo mismo del resto de soportes con los que se comunican. Sin embargo, hasta hace poco, la televisión parecía refractaria a esta demanda del público. Aún más, propuestas como la Televisión Digital Terrestre, ofrecidas al público como el definitivo advenimiento de la televisión interactiva, no han sido capaces de satisfacer la necesidad de participar, personalizar y alterar el tradicional esquema emisor-receptor.

Por tanto, los prosumidores de Toffler (1984) han decidido que, definitivamente, también la televisión debe ser un medio en el que tomar parte activamente, superando el modelo de espectador pasivo. Soluciones como las aplicaciones second screen (segunda pantalla), o la natural sociedad de redes sociales y programas de televisión, han sido la primera forma de encauzar ese impulso de las audiencias por participar de la experiencia televisiva. De hecho, el uso de redes sociales y el consumo de contenidos audiovisuales ha ido gradualmente solapándose (Ludányi y Belényasi, 2011).

A ese tipo de soluciones que han dado forma a la televisión social (Harboe , 2009: 724-731; Steffen, 2012: 7 y 10) se unen ahora tecnologías pensadas para ofrecer por fin esa interactividad que el usuario busca. Llegan con esas propuestas las llamadas televisiones conectadas, inteligentes o smart tv. Se trata de dispositivos con conexión a Internet que permiten la interacción con contenidos en línea no necesariamente vinculados a la emisión televisiva.

En poco más de cinco años, el estándar Hybrid Broadcast Broadband Television, también denominado Televisión Digital Híbrida, TDT Híbrida o, más habitualmente, HbbTV, se ha extendido entre los fabricantes de televisiones. De acuerdo con 
Strzebkowski, Bartoli y Spielvogel (2011), hay un “evidente crecimiento" de la infraestructura de smart tv o televisiones conectadas capaces de ejecutar aplicaciones interactivas en una pantalla hasta hace poco unidireccional. Los fabricantes de televisiones apuestan por el desarrollo de tecnologías que amplifican la experiencia de los telespectadores, llevándolos de este estado al de usuarios (Mansilla y Marcos, 2013).

La propuesta de conexión tiene dos expresiones mayoritarias. Por un lado, un enfoque similar al de Apple y otros fabricantes de hardware y software, que apuestan por la distribución de contenidos a través de aplicaciones de código propietario que se instalan en los televisores (gratuitamente o previo pago), o que llegan con los aparatos como parte de su dotación original de serie.

Por otro, el enfoque HbbTV, en el que se establece una conexión entre el canal y programa que se ven en la televisión (sean lineales u on demand) y una aplicación HbbTV interactiva con contenido adicional al del programa que se está viendo (u otros contenidos) y que es ofrecido on line (Strzebkowski, Bartoli y Spielvogel, 2011).

Por tanto, las plataformas HbbTV proponen un acceso a Internet que no precisa de descargas de software por parte de los usuarios. Tampoco es necesario utilizar ningún programa específico preinstalado en el televisor. Simplemente, los receptores son capaces de leer, decodificar y mostrar lo que reciben on line gracias a la estandarización de los datos, que es la base del HbbTV. Para leer y plasmar la información entrante, la televisión solo ha tener un navegador capaz de reproducir información codificada como HTML.

Así, el estándar HbbTV (http://www.HbbTV.org/) es una norma de conexión de televisiones a servicios que corran sobre tecnología IP. De acuerdo con Fondevila (2013: 15), el HbbTV “aglutina partes de distintos estándares ya creados, permite sincronizar el entorno de broadcasty broadband”. Es decir, supone la creación de una plataforma que, desde la televisión, permite recibir emisiones televisivas y también contenidos on line.

\subsection{Estado del arte del HbbTV}

El HbbTV se remonta a 2009, cuando un grupo de fabricantes, broadcasters y otras entidades europeas acordaron la creación de un modelo único y abierto de distribución de contenidos on line que pusiera al consumidor ante una "experiencia holística" (Fondevilla, 2013:14) de entretenimiento e información.

Actualmente, el proyecto aglutina más de 60 empresas, principalmente europeas, y trabaja en nuevas evoluciones del estándar, que en 2012 alcanzó la versión 1.5, que llegará en 2014 a los nuevos modelos de televisores ${ }^{1}$. El grupo de miembros directores del consorcio está compuesto por Abertis Telecom, ANT Software, Digital TV Labs, European Broadcasting

1 Para conocer en detalle las especificaciones técnicas del estándar HbbTV en su versión 1.5, se recomiendo consultar este recurso: http://www.HbbTV.org/pages/about_HbbTV/HbbTV-specification-1-5.pdf 
Union, France Television, Institute für Rundfunktechnik, Open TV, Opera, RTL Group, Samsung, SES Astra, Sony Corporation, Television Francaise 1 - TF! Y TPVision.

El estándar se encuentra en su versión 1.5. Con esta versión, liberada en 2012, se despliegan nuevas funcionalidades que podrán ser construidas sobre desarrollos HbbTV y que los navegadores anteriores no soportaban. Por ejemplo, se podrán realizar emisiones de streaming en directo al margen de la emisión lineal u on demand que se consuma. Así, un usuario/espectador podrá ver un programa y, simultáneamente, una emisión en streaming y en directo -live-que puede estar vinculada o no con el contenido que ve (ITU, 2013: 4).

Este nivel de desarrollo permite construir páginas reproducibles por los navegadores o browsers de las televisiones. Se construyen en el lenguaje CE-HTML, estándar de desarrollo para dispositivos de electrónica de consumo y aprobado por el consorcio W3C Web, que establece los estándares de construcción web. Soporta también Java-Scripts (ECMAScript262), XHTML 1.0, CSS TV y DOM2.

Las páginas así construidas pueden mostrar texto, imágenes fijas y también vídeos y audios, además de, como hemos visto arriba, vídeo en streaming. En este caso, el estándar 1.5 soporta streaming adaptativo (basado en la tecnología MPEGDASH), que "mejora la calidad percibida en las presentaciones de vídeos con conexiones a Internet lentas o saturadas" (ITU, 2013:11).

En términos de desarrollo web, las posibilidades visuales y funcionales resultan un tanto anticuadas y equivaldrían a los niveles constructivos de las páginas de mediados de la década pasada, pensadas para navegadores como Explorer 5 y 6 . Se debe a que los procesadores y navegadores que llevan las televisiones no tienen la capacidad de trabajo de los procesadores convencionales de la industria de los ordenadores personales. Por esta razón, el trabajo de programadores, diseñadores e informadores se ve constreñido por todas estas limitaciones técnicas, que impiden el desarrollo de funcionalidades y soluciones frecuentes en otros entornos, como ordenadores domésticos o incluso tabletas electrónicas.

Otra limitación muy importante es que la navegación se realiza a través del mando a distancia de la televisión como única vía de interacción entre usuario/espectador y el servicio HbbTV. Por un lado, esta limitación es una cierta ventaja, puesto que incorpora a Internet a públicos que, habituados al uso del mando a distancia, no utilizan habitualmente ordenadores u otros dispositivos. Para estos usuarios, el mando de la televisión es una tecnología más conocida que los aparatos informáticos, con lo que se generan menos resistencias.

Pero también constituye un problema a la hora de construir las páginas del servicio, puesto que la navegación está acotada por los controles del mando: botones de colores (rojo, amarillo, azul y verde), teclado alfanumérico y cursores. Por este motivo, la arquitectura de información y contenidos, y toda la navegación, han de estar pensadas para ser ejecutadas utilizando solo esos controles, y sin recurrir a más periféricos. 
Existe, sin embargo, la posibilidad teórica de coordinar el sistema HbbTV con una aplicación para tableta o teléfono móvil inteligente, que podría hacer las veces de mando a distancia más funcional y completo. Los experimentos de Strzebkowski, Bartoli y Vogel (2012) inciden en ese tipo de integración, añadiendo, además, el uso de tecnologías como el lenguaje estándar HTML 5 en lo que denominan "nueva generación de HbbTV". Este tipo de soluciones podrían dotar a los desarrollos HbbTV de funcionalidades más actuales y, sobre todo, darían al usuario una capacidad de interacción que hoy no tiene. Sin embargo, suponen la introducción de un elemento tecnológico -tableta o teléfono inteligente- que puede suponer una barrera de manejo para públicos no habituados a estos dispositivos.

\subsection{Experiencias de servicios HbbTV}

En cuanto a desarrollos de experiencias HbbTV, hay una cierta colección a nivel mundial, si bien hay que destacar que se echan en falta planteamientos pensados desde el origen para este tipo de soporte. La mayoría de las propuestas trasladan al entorno HbbTV productos web adaptados. O, en todo caso, repositorios de vídeo para su consumo on line.

La primera experiencia de emisión de contenidos HbbTV tuvo lugar en Francia en el año 2012, cuando France TV llevó a cabo una demostración de esta tecnología aprovechando la retransmisión del torneo de tenis Roland Garros (Panorama Audiovisual, 2012). En ese mismo año, la celebración de los Juegos Olímpicos de Londres serviría como hito de lanzamiento para otros muchos servicios de este tipo, como, por ejemplo, la primera propuesta HbbTV de TVE (RTVE.es, 2012).

En un informe de la International Telecommunications Union (2013: 10) se citan algunas de las propuestas más destacadas de las que se han hecho hasta ahora, como las de France 24, la alemana ARD, la holandesa NPO y la de la cadena española Telecinco, si bien este último servicio ha dejado de estar operativo, a la espera de que el grupo Mediaset presente nuevas soluciones en esta línea.

Además de la ya citada experiencia de Televisión Española para los Juego de Londres 2012, la cadena pública presentó en 2012 su desarrollo HbbTV para contenidos audiovisuales infantiles (Clan). Y, desde entonces, en España se han desarrollado otros servicios, como los de las cadenas Gol TV, Veo, TV3, Telemadrid o EiTB². En todos los casos se trata de traslaciones a HbbTV de contenidos ya existentes en las webs de estas empresas.

\subsection{Influencia sobre el ecosistema televisivo}

La irrupción de la tecnología HbbTV está llamada a ejercer una notable influencia sobre el ecosistema de los contenidos televisivos. Siguiendo la metáfora de la "ecología mediática" (Scolari, 2010), la televisión se defiende del acoso de otros entornos, como las redes sociales de Internet, que roban tiempo de consumo televisivo a los espectadores y usuarios.

2 Para mayor detalle de los servicios HbbTV en España, consultar la web de la TDT Híbrida: http://www.tdthibrida.es/index.php 
El mismo Scolari (2013) propone que la televisión “imita a los medios digitales interactivos” en su propuesta fragmentada, acelerada y de narrativa deconstruida. Sin embargo, sobre un soporte como la televisión digital híbrida conectada, se supera la idea y la televisión absorbe parte de las capacidades de esos medios digitales, con lo que logra recuperar tiempo frente al resto de medios. Esta aportación es muy importante en un momento en el que la pugna por la atención del público es la clave de un mercado saturado en todos los soportes por una casi infinita oferta tanto informativa como de entretenimiento.

La televisión habría iniciado de esta forma una incipiente reconquista del territorio en la lucha por la atención de los espectadores/usuarios. Y no solo a través de herramientas habituales de los cibermedios, sino compitiendo con ellos en plataformas novedosas, como las redes sociales.

La industria de la televisión (broadcasters, creadores de contenidos, anunciantes y también fabricantes de equipos) es consciente de la importancia del fenómeno de la televisión social, que se ha impuesto en los últimos años como nueva forma de consumo de productos televisivos y que entusiasma a buena parte de la audiencia, sobre todo la más joven.

Eduardo Prádanos (2011) describe esta televisión social como "la tecnología de televisión que permite y fomenta la interactividad entre espectadores”. Es decir, ver la televisión se torna una experiencia más social que nunca, pero trasciende a la inmediata presencia de los televidentes que comparten espacio físico y se convierte en una experiencia compartida, ubicua y expansiva (Jonietz, 2010; Merino, 2013; Castelló, 2013; Castillo, 2013). De hecho, la conjunción de televisión y redes ahonda en la comprensión de los espectadores/usuarios en tanto que prosumidores (Stefanone, Lackaff yRosen, 2010: 520).

Irrumpiendo en este complejo panorama de la televisión social, HbbTV (y otras vías de televisión conectada) supone la introducción de variables interesantes por su condición de tecnología puente entre otras muy extendidas. Con su versatilidad, la televisión conectada permite a estas audiencias sociales mantener sus prácticas de intercambio y compartición desde aparatos que, físicamente, estaban al margen de estas prácticas. La posibilidad de conectarse con herramientas de redes sociales, como Facebook o Twitter, desde el televisor es un paso más en la forma de consumir tanto Internet como la propia televisión. Se logra, además, con una tecnología considerada "neutral" (Fondevila, 2012:15) y dando lugar a lo que Vilches (2013: 25) considera una "parada estratégica para el futuro de ambos medios".

$\mathrm{Si}$, como se ve, las bases tecnológicas están asentadas, no podemos decir lo mismo de la experiencia de usuario, es decir, la relación entre el usuario, el interfaz y las funcionalidades que este ofrece. En el caso de la tecnología HbbTV, hay que señalar que la experiencia de usuario es todavía bastante pobre. Está muy condicionada por el espacio donde se produce la interacción, que es la televisión gestionada con el mando a distancia. Los problemas de experiencia son una amenaza (Fondevila, 2012: 16; Mansilla y Marcos, 2013: 127) y un desafío para tecnólogos, fabricantes, desarrolladores, arquitectos de información e informadores. 
Estos últimos están también involucrados en otra de las fallas que aquejan al HbbTV: la falta de producción de contenidos pensados para este soporte. De acuerdo con Cardoso (2011: 203), la innovación en contenidos es “escasa”. Y, de hecho, como veíamos en las experiencias de HbbTV, la mayoría de las iniciativas se centran en trasladar a las televisiones versiones de lo que se puede encontrar en otras pantallas. Faltan estrategias de desarrollo de contenidos planteados desde el origen para ser encontrados y consumidos en plataformas de HbbTV.

\subsection{RTVE: apuesta por el Botón Rojo}

Ante esta falta de propuestas de contenidos pensados para la televisión digital híbrida, resulta relevante la iniciativa de Radio Televisión Española denominada RTVE Botón Rojo, primer intento sólido de construir un servicio de información pensado exclusivamente para HbbTV.

La Corporación RTVE, empresa pública, despliega desde 2007 una estrategia multimedia y digital que la ha situado a la cabeza de la investigación en nuevos formatos en España, y que la ha alineado con las principales radiotelevisiones europeas (Medina y Ojer, 2011: 93).

Esta estrategia de desarrollo de nuevos medios ha llevado a RTVE a contar con un ecosistema digital que se apoya en la web www.rtve.es y en un amplio abanico de aplicaciones para teléfonos inteligentes, tabletas digitales, consolas de videojuegos y televisiones conectadas, tanto a través de software propietario (aplicaciones), como en la modalidad HbbTV.

Organizado en torno al departamento de Medios Interactivos, este despliegue supone la presencia de RTVE y sus contenidos de televisión, radio e Internet en prácticamente todos los ámbitos del mercado digital actual. Al tiempo, la estrategia de RTVE se ha desarrollado ampliamente sobre las redes sociales de Internet, construyendo comunidades de intereses y personas en torno a sus contenidos y construyendo experiencias de televisión social, radio social y participación de la audiencia.

Ya se ha visto arriba que RTVE puso en marcha sus experimentos de HbbTV en 2012, durante los Juegos Olímpicos de Londres (Figura 1). En aquel momento se configuró el acceso a través de las televisiones a un portal de información deportiva en el que se podían ver las pruebas olímpicas y recibir datos de resultados deportivos. 
Figura 1. Portal HbbTV de RTVE en 2012

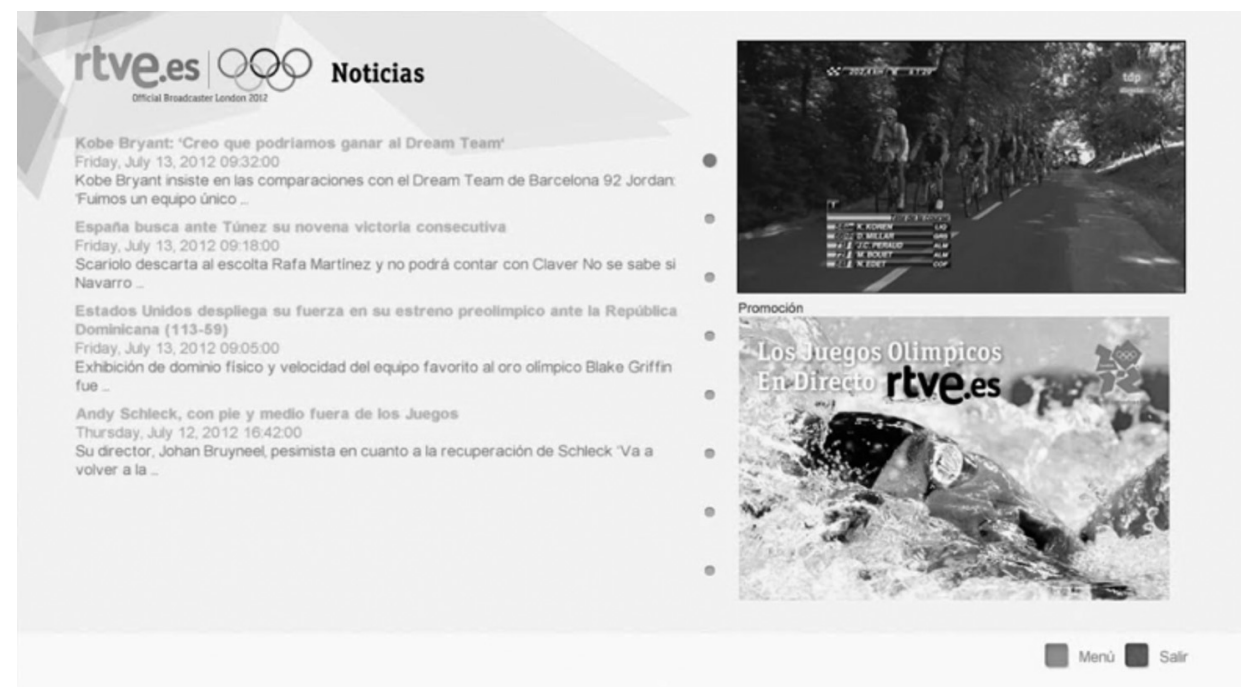

Fuente: Elaboración propia, tomado de RTVE

A esta primera propuesta de 2012 siguieron otras dos que tomaron el relevo tras la cita olímpica: RTVE a la Carta y Clan. En ambos casos, la plataforma HbbTV permitía la visualización de contenidos 'a la carta', es decir, vídeo on demand. Los usuarios podían navegar por repositorios de vídeos y reproducir aquellos que les interesaban en sus televisiones, ejecutándolos directamente a través de un player on line.

Las tres propuestas iniciales, pese a haber constituido un paso decidido en la implantación de la HbbTV en España, se quedaban una vez más en la traslación de contenidos ya disponibles en la web de RTVE al nuevo soporte.

Sin embargo, en 2013 RTVE, a través del departamento de Medios Interactivos, da un salto de calidad al presentar un nuevo servicio HbbTV que, además de mostrar elementos ya presentes en su web, ofrece, por primera vez, contenidos específicamente creados para ser consumidos en un entorno de smart t $v$.

El servicio se denomina RTVE Botón Rojo y marca un punto de inflexión en el desarrollo de esta tecnología en España. Cabe esperar que la iniciativa de RTVE arrastre al sector, estimulando la aparición de servicios similares, lo que seguramente supondría una buena noticia para el periodismo, la industria televisiva en general y para los usuarios/espectadores.

Al tiempo, RTVE busca nuevas audiencias a las que llevar sus contenidos. Con las televisiones conectadas, se incorporan a Internet bolsas de público que están al margen de la sociedad digital, bien por desinterés en la informática, bien porque 
las tecnologías de la comunicación y la información suponen una barrera de acceso que la televisión, más familiar y conocida, no presenta.

De esta forma, se contribuye a cerrar un tanto la brecha digital y se ofrece una vía de acceso a la información y el entretenimiento digitales a personas que no contaban con estas fuentes. Son públicos que, a través de estos medios, se podrán incorporar a entornos como las redes sociales o a los fenómenos de empoderamiento que las nuevas tecnologías propician.

El mercado, por su parte, muestra claro interés en la nueva tecnología y en su capacidad de impulsar una renovación en el parque de televisiones. Un informe de la Interactive Advertisign Bureau para España (IAB, 2013) señala que un 86 por ciento de los encuestados sabe qué es la televisión conectada. Sin embargo, solo un 18 por ciento declara tener ya un aparato conectado. El trabajo calcula un parque de televisiones susceptibles de conectarse a la red de unos tres millones de unidades. Sin embargo, IAB considera que solo un 36 por ciento está conectado.

\section{Material y métodos empleados}

Para llevar a cabo este trabajo se han utilizado tres técnicas en combinación. Al ser el autor miembro del equipo de RTVE que ha desarrollado el servicio HbbTV, se cuenta con una suerte de observación participante (Alzate, 2011; Gaitán y Piñuel, 1998), si bien orientada de forma diferente a la que los autores describen. La posibilidad de participar en todo el proceso permite elaborar una bitácora del proyecto que, a modo de notas de campo, se convierte en uno de los instrumentos fundamentales para comprender el objeto de investigación y su proceso de conceptualización, diseño, construcción y lanzamiento.

A este método de trabajo se unen las conversaciones (Gaitán y Piñuel, 1998) mantenidas con responsables del proyecto como parte del propio proceso de trabajo de RTVE y con fines aclaratorios o para ampliar aspectos necesarios en esta descripción formal.

Estas técnicas se combinan con el análisis de un juego de datos estadísticos oficiales procedentes del equipo de monitorización y márketing de RTVE. Estos datos, como es obvio, son propios de la compañía. Esto supone una variación en la medición habitual de tráficos on line, que utiliza cálculos externos, auditados o elaborados por terceros. Sin embargo, las condiciones de desarrollo incipiente de la tecnología no permiten este tipo de mediciones. Los televisores conectados no guardan cookies-ficheros de reconocimiento de usuarios-, ni tienen otros sistemas habituales de medición, como los que emplean empresas como Nielsen, Comscore u otras especialistas en monitorizacón. Así, es prácticamente imposible establecer mediciones exhaustivas y tan fieles como las habituales en el entorno web. Por este motivo, no queda otro camino que trabajar con las mediciones internas de las empresas desarrolladoras que, si bien tampoco permiten alcanzar niveles de detalle y precisión ideales, se aproximan y ofrecen vistas del fenómeno estudiado que no son dadas a agentes externos. 
Con este material, se ha configurado un análisis distribuido en dos partes. De un lado, se ha llevado a cabo una descripción formal del producto denominado Botón Rojo de RTVE, expresión final del proyecto de HbbTV de RTVE. Se ha tratado de explicar qué elementos componen el servicio y cuál ha sido el camino que se ha seguido para configurarlos, desde la fase de concepto hasta la puesta en línea definitiva.

De otro lado, se ha realizado un análisis de los datos estadísticos para comprender en qué medida el Botón Rojo está siendo utilizado por el público y con qué interés. Se ha tratado así de establecer parámetros de comportamiento y uso por parte de los espectadores y usuarios.

Con estas herramientas metodológicas se ha buscado aportar una visión relevante sobre un proyecto que, por su carácter pionero, se considera que ha establecido un punto de partida en el devenir de este nuevo soporte, y también en la configuración de futuras rutinas profesionales para diseñadores, programadores y periodistas.

\section{Análisis y resultados}

Se afronta por tanto la primera parte del análisis trazando la descripción formal del servicio de Botón Rojo de RTVE, atendiendo, sobre todo, a su arquitectura de contenidos informativos y de entretenimiento. Es decir, a la vista que el usuario tiene en su televisión y a las posibilidades de interacción con esa arquitectura y sus contenidos.

\subsection{El proyecto Botón Rojo de RTVE}

La Corporación RTVE decidió intensificar su presencia en la televisión digital híbrida en abril de 2013. En ese momento, el departamento de Medios Interactivos asumió el encargo de poner en marcha un nuevo servicio HbbTV que complementase el ya disponible desde 2012.

La fase de conceptualización se inició a principios de mayo de 2013 y en ella tomaron parte un equipo multidisciplinar de RTVE y la empresa de diseño londinense Audacity Partners. A lo largo de varias sesiones, diseñadores, desarrolladores y periodistas colaboraron para dar forma al servicio. En esta etapa, el esfuerzo creativo resultó determinante, puesto que se trataba de configurar un nuevo tipo de acceso a la información de una forma que apenas cuenta con referentes en el ecosistema televisivo.

Finalmente, el equipo de trabajo propuso la construcción de un portal en tecnología HbbTV con dos vías de consumo. Por un lado, una zona de acceso permanente a contenidos informativos y de entretenimiento. Por otro, una capa interactiva de información no permanente confeccionada 'ad hoc' para ser lanzada y consumida simultáneamente a determinadas emisiones televisivas. 
La primera zona -información permanente- responde a una idea convencional de consulta de Internet a través de un acceso similar al que puede ofrecer cualquier portal de la red, pero en la televisión. En su inicio, se concibe como un portal de acceso a noticias, resultados deportivos, vídeos de RTVE.es A la Carta y vídeos de Clan, la web infantil de RTVE.

Conceptualmente, estamos ante una vía de consultar noticias a través de un aparato nuevo en la conexión con la información en línea: la televisión. De hecho, como hemos visto, el acceso a los servicios de A la Carta y Clan ya estaba disponible en 2012, pero no las noticias. En esta nueva etapa se ha mejorado notablemente su apariencia, y se ha rodeado del mencionado servicio de noticias, tanto de información general como deportivas, además de resultados deportivos actualizados en tiempo real.

Lo que se define como capa interactiva de información no permanente tiene más interés desde el punto de vista del concepto de televisión conectada y desde la óptica de la convergencia de medios. También resulta más valioso como revulsivo en el uso de la televisión y como herramienta de atracción de nuevos públicos. Esta parte del servicio de Botón Rojo se concibe como un conjunto de eventos informativos que se crean como acompañamiento de la emisión televisiva, de forma que la complementan, expanden y enriquecen.

Estos eventos son pequeñas piezas informativas y pueden ser textos, imágenes fijas, vídeos, juegos de pregunta-respuesta, encuestas, etc. Su principal característica es que no están siempre disponibles, sino que su “vida útil” está ligada a la emisión televisiva y no tienen sentido al margen de esta. Así, son complementos on line de la emisión de televisión digital terrestre, aunando en una sola experiencia la visualización de contenidos televisivos con la interacción virtual con contenidos habitualmente solo disponibles en Internet.

\subsection{Implementación y el lanzamiento}

Como en cualquier desarrollo de HbbTV, el principal inconveniente para el equipo de RTVE en la construcción del nuevo servicio de Botón Rojo resultó ser la disparidad de la experiencia de usuario. Esto se debe a que cada fabricante de televisiones utiliza procesadores y navegadores ('browsers') diferentes, con lo que, aunque todos dan acceso a los contenidos, no todos los muestran en pantalla de igual forma. Esto exige un complejo trabajo de ajuste para los desarrolladores y para los diseñadores, que persiguen una experiencia uniforme para todo el servicio. Las tareas de ajuste de la maquetación son imprescindibles para uniformizar esa relación de los usuarios con el portal.

El segundo escollo es la complejidad que supone construir una navegación que se gestiona con un mando a distancia como herramienta única de interacción. Acostumbrados a la facilidad que ofrecen herramientas como el teclado o el ratón, o, aún más, la sencillez de manejo de los dispositivos hápticos, los equipos de desarrollo se ven muy limitados a la hora de plantear cualquier acción en el portal HbbTV: lo que en otros entornos se soluciona de forma muy simple a través de he- 
rramientas muy versátiles, en este nuevo entorno exige un replanteamiento y una reflexión profunda. De esta forma, el equipo de RTVE tuvo que simplificar las acciones posibles sobre el portal, hasta dejarlas en un mínimo: subir, bajar, navegar a izquierda y derecha (cursores del mando), entrar a contenidos (botones de colores y botón ok del mando) y seleccionar elementos en un menú (utilizando los números del mando).

En esta simplificación fueron descartadas todas las opciones que pasaban por introducir textos en el sistema a través del mando, lo que descarta de antemano cualquier posibilidad de hacer log-in y de personalizar la experiencia, algo que se planteó en un primer momento pero que fue descartado por los inconvenientes de experiencia de usuario descritos.

Finalmente, una complejidad adicional es la propia naturaleza del servicio propuesto. Al tener que coordinar la capa de información adicional con la emisión televisiva, fue preciso desarrollar un sistema de sincronización entre los sistemas de emisión de Televisión Española y el gestor de creación de contenidos on line. Este desarrollo, se llevó a cabo de forma que sirviera también para crear contenidos disponibles en otros servicios de RTVE, como la aplicación de second screen +TVE (La Vanguardia, 2013).

Una vez construido, el nuevo portal HbbTV fue lanzado públicamente el día 5 de septiembre de 2013, reemplazando definitivamente al anterior servicio. Desde ese día, los usuarios y espectadores de televisiones conectadas pueden acceder al Botón Rojo utilizando, precisamente, el botón rojo de su mando a distancia, que conecta los elementos interactivos y sirve de llave al portal.

Así, las televisiones capaces de conectarse muestran periódicamente avisos que recuerdan al espectador esta posibilidad. Si el usuario/espectador quiere activarla, tiene que pulsar el botón rojo, lo que invoca una capa interactiva que hace las veces de menú. Esta capa interactiva (Figura 2) es navegable con los cursores del mando a distancia (izquierda y derecha), y también con los botones de colores del mando. El botón de color amarillo da acceso directo a los contenidos A la Carta; el azul, a la zona de noticias, mientras que el botón verde permite acceder a la información deportiva. Los contenidos infantiles de Clan son recuperables navegando con los cursores y el botón de Ok del mando: no tienen un color asignado. 
Figura 2. Menú de inicio del portal HbbTV de RTVE

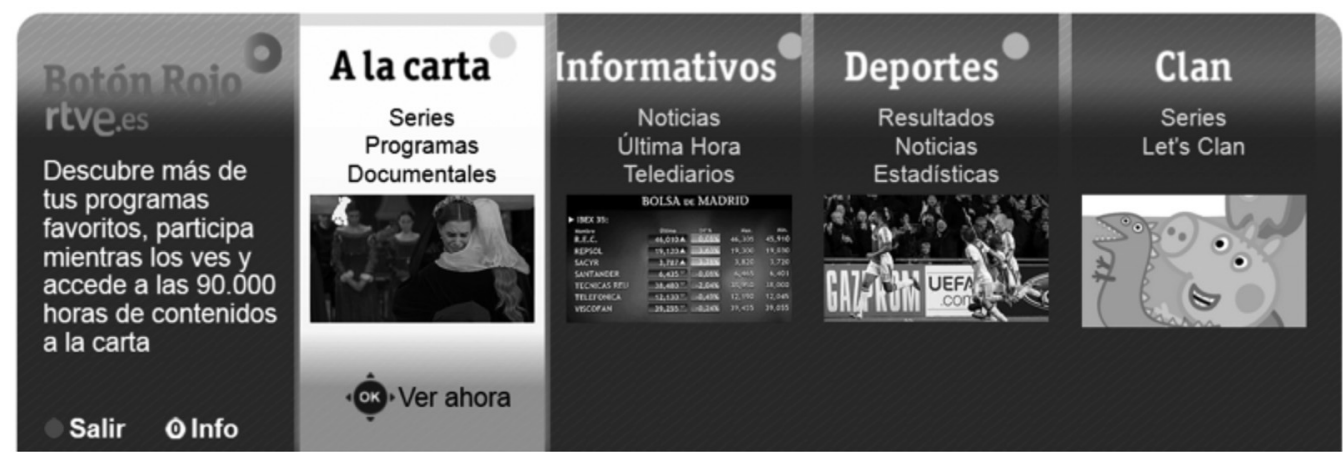

Fuente: Elaboración propia, tomado de Botón Rojo de RTVE.es

Acceda navegando con los cursores o con los colores, el usuario -aquí ya no cabe considerarlo simplemente espectadorse encuentra en un entorno que recuerda al que se puede encontrar en cualquier web informativa: páginas de texto, imágenes y vídeos a través de las cuales puede desplazarse utilizando los cursores. La parte superior de la página permite navegar entre las diferentes secciones. Si se desciende un paso en la arquitectura, se navega entre noticias, mientras que la parte inferior de la página muestra vídeos de programas informativos de Televisión Española. Para cargar una noticia completa o reproducir un vídeo, basta con pulsar el botón Ok del mando.

Después, la noticia se puede navegar hacia abajo con los cursores, y se puede saltar hacia la siguiente noticia utilizando los botones de desplazamiento lateral, generalmente marcados en los mandos con iconos de flechas. Es decir, el paso entre secciones y contenidos se hace siempre subiendo o bajando, y desplazándose lateralmente con los cursores del mando. Desde cualquier sección se puede saltar a otra a través de los botones de colores, o volviendo con los cursores a la parte superior de la página y navegando a través de los enlaces. 
Figura 3. Aspecto de la zona de Noticias en HbbTV de RTVE

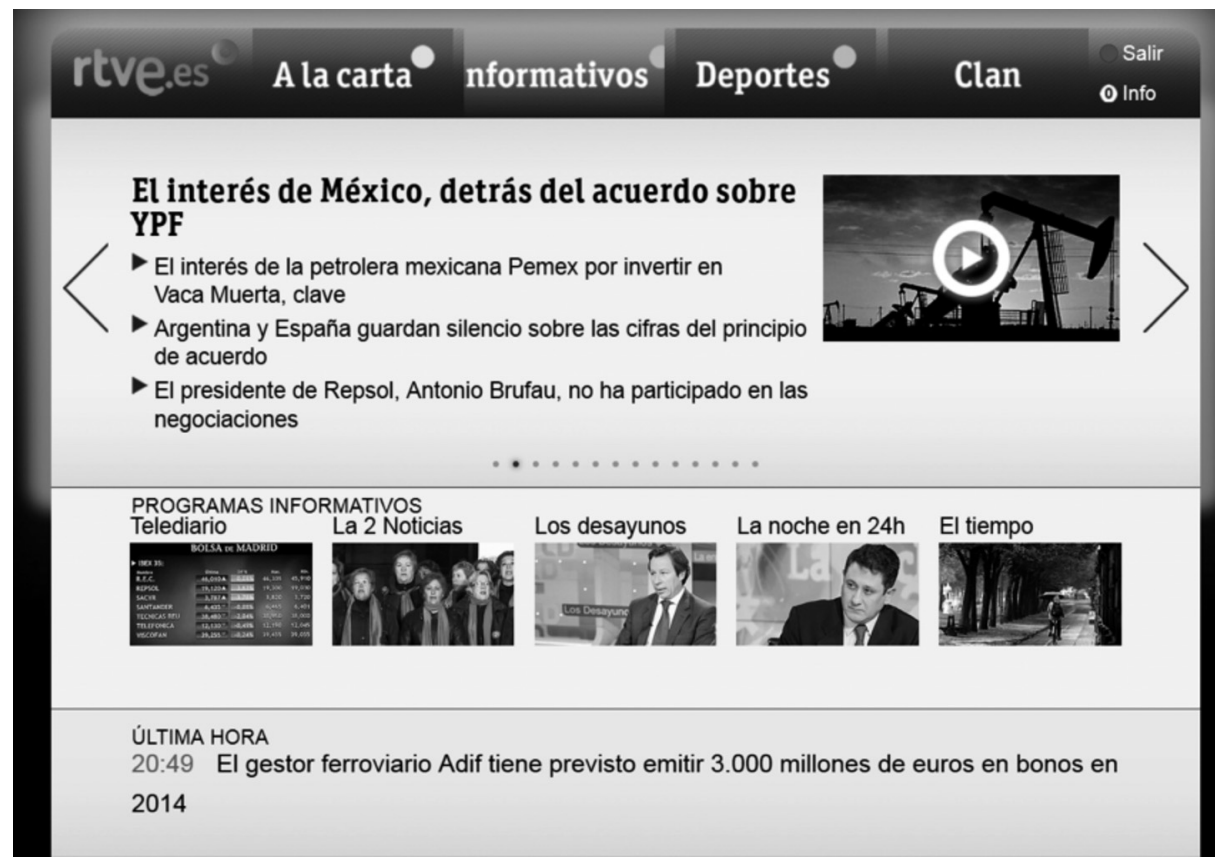

Fuente: Elaboración propia, tomado de Botón Rojo de RTVE.es

En la sección de Deportes, marcada con el color verde, la arquitectura de navegación es idéntica, con la diferencia de que la parte inferior de la página no muestra vídeos, sino acceso resultados de competiciones deportivas. Al ir profundizando en estos resultados, se muestran tablas clasificatorias, calendarios de competiciones y resultados de encuentros de fútbol, baloncesto u otros deportes.

Por su parte, la capa de interactividad sincronizada con la emisión no se puede invocar en cualquier momento, sino que el sistema lanza sobre la emisión un aviso que informa al espectador/usuario de que el contenido que está viendo tiene disponible algún tipo de información suplementaria. Basta con pulsar el botón rojo para recibir esa información, que aparecerá a medida que la sincronía establecida por los productores de contenidos lo requiera.

Así, en un momento concreto, un mensaje emergente alertará de nuevo de que se puede acceder a un contenido. Con el botón rojo, el usuario/espectador despliega ese contenido extra como una capa de contenido sobre la emisión televisiva. De esa forma, televisión e Internet conviven en un mismo espacio, permitiendo al usuario la consulta simultánea de ambas ofertas. Esta forma de ver la televisión es completamente innovadora, puesto que, hasta ahora, los elementos 
no televisivos que en algún momento irrumpían sobre las emisiones, no solían ser interactivos (excepto en los casos de plataformas de pago).

Como hemos visto antes, el contenido que se lanza puede componerse de textos, cifras, imágenes o elementos interactivos como preguntas o encuestas. Una vez terminada la emisión, estos suplementos dejan de estar accesibles.

Lo novedoso en la propuesta es que estos contenidos extra sincronizados se crean 'ex profeso' para el servicio HbbTV, no son necesariamente la traslación de otros elementos ya presentes en la web. De esa forma, el servicio se estrenó con el Telediario de La 1 de TVE, cuya emisión es enriquecida con información que complementa on line las noticias audiovisuales. Este trabajo es llevado a cabo por los redactores del Telediario, que, editorialmente, deciden qué parte de las informaciones se destina a esta capa complementaria y en qué momento se lanza cada nuevo contenido.

Además, para enriquecer otras emisiones (programas, deportes o series), RTVE ha creado un nuevo equipo de cuatro redactores. Son los encargados de decidir qué emisiones cuentan con este añadido y qué tipo de contenidos se crean.

Figura 4. Información simultánea a una emisión televisiva

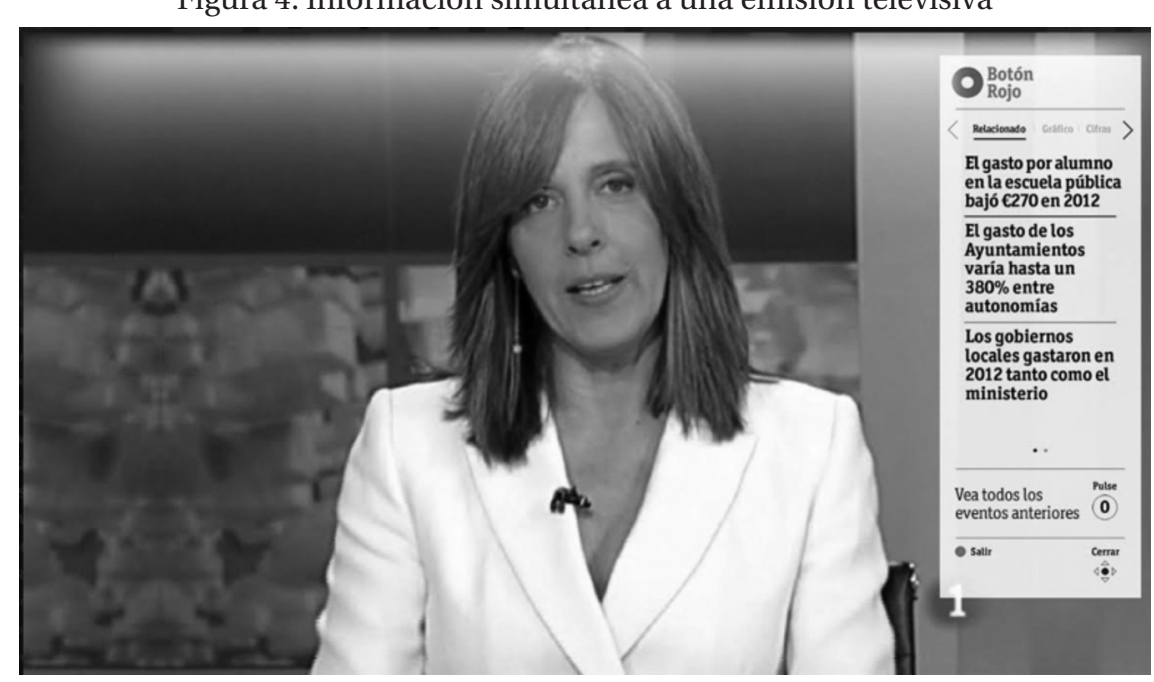

Fuente: Elaboración propia, tomado de Botón Rojo de RTVE.es

\subsection{Primeros resultados}

Ya se ha mencionado antes la dificultad que entraña la medición del tráfico 'on line’ en las televisiones, aparatos poco dotados para esta tarea de medición. Por eso, los datos con que se cuenta son mediciones internas de RTVE hechas a través 
de tags de marcado insertos en el código HTML que compone el portal y sus diferentes elementos. De esta forma, se pueden medir algunos parámetros, pero sin la fiabilidad y amplitud que acostumbra la industria de Internet.

Lanzado el 5 de septiembre de 2013 (Europa Press, 2013), el servicio de Botón Rojo acompañó su estreno con una campaña en televisión, radio, webs y redes sociales. La repercusión de la campaña tuvo efecto inmediato en la llegada de nueva audiencia a la plataforma de HbbTV de RTVE.

Si antes del lanzamiento se recibían unos 110.000 visitantes únicos semanales, los datos de RTVE indican que la semana de la presentación la cifra llegó a los 269.406, número que creció en las semanas posteriores para llegar al entorno de los 600.000 a finales de octubre de 2013.

Este crecimiento en las visitas permite hablar de una favorable acogida por el público, que encuentra más atractiva la oferta de RTVE en HbbTV y, por tanto, dedica más tiempo y esfuerzo a explorar el portal y todo el Botón Rojo.

Por contenidos, los vídeos infantiles son las piezas más buscadas del servicio. Su demanda es muy alta y concentra aproximadamente un $40 \%$ del tráfico, con una media cercana a 20.000 usuarios diarios (datos de la semana del 11 al 18 de noviembre de 2013), manteniendo una tendencia al alza en comparación con las semanas previas.

Por detrás de los contenidos infantiles aparecen los de A la Carta, que son programas y series de las diferentes cadenas de TVE. En la semana del 11 al 18 de noviembre, por ejemplo, A la Carta recibió una media de 10.000 visitantes diarios, de nuevo con tendencia al alza frente a las series temporales anteriores. Las nuevas ofertas informativas, noticias generales e información deportiva muestran cifras más bajas, lo que indica que el público apuesta por la interactividad para recuperar contenidos televisivos que no ha podido ver, o que quiere volver a ver, y todavía no muestra demasiado interés por la información en este soporte. Sin embargo, de nuevo los números indican incrementos de semana en semana, y, paulatinamente, más usuarios eligen esta vía para informarse.

Dentro de este crecimiento, el interés por el deporte es más relevante: las noticias y los resultados deportivos son el tercer bloque de contenidos para los usuarios, por encima de la oferta de información general.

Resulta necesario analizar el momento de acceso, toda vez que la televisión no es un objeto portátil. Así, los datos muestran con claridad que el uso de los servicios HbbTV es paralelo al uso de la televisión tradicional, ajustándose a los llamados 'prime time'. Se observa un primer lapso entre las 14:00 y las 16:00, la hora del almuerzo en España, cuando los usuarios/espectadores comen y aprovechan para ver la televisión, especialmente contenidos informativos. Después, la curva de audiencia mantiene vigor durante la tarde, pero toma fuerza a partir de las 19:00, cuando ya los niños reclaman contenidos infantiles. 
La comparación de la curva de audiencia general por horas con la del consumo de vídeos infantiles por horas demuestra que ese momento, las 19:00, concita el mayor tráfico en los días laborales. A partir de las 20:00 horas, el tráfico decae, encontrando otro punto importante, aunque de menor relevancia, en torno a las 22:00.

El comportamiento en fines de semana es parejo, con la diferencia de que tanto los contenidos generales como los infantiles encuentran demanda por la mañana, sobre las 10:00 horas. Se aprecia, además, que en los fines de semana las tardes tienen más usuarios adultos, puesto que hay más demanda de contenidos generales que en los días laborables.

Por otra parte, conviene analizar por separado el uso de la información interactiva simultánea, que es probablemente la parte más innovadora y arriesgada, del proyecto de RTVE. Los datos de los primeros dos meses muestran un creciente interés por parte del público en este contenido interactivo.

Sin ser todavía un acceso masivo, se detecta una tendencia de crecimiento en el uso de la información interactiva que acompaña a determinados programas, como los Telediarios. Por tomar una referencia, en la semana del 11 al 18 de noviembre, los contenidos complementarios recibieron un $17 \%$ más de visitas que en la semana anterior, lo que indica que el ritmo de descubrimiento de esta alternativa de información es constante.

Desde el lanzamiento, cada semana son más los usuarios que invocan estas piezas informativas 'on line' que acompañan a las emisiones lineales de televisión, lo que parece indicar que resultan interesantes o útiles a los usuarios/espectadores.

En cuanto al momento de mayor uso de esta opción, coincide con la emisión del Telediario de la noche de TVE, entre las 21 y las 22 horas. Los telediarios de las 15:00 horas también registran uso, pero en mucha menor medida.

\section{Discusión y conclusiones}

El análisis del servicio de Botón Rojo de RTVE arroja algunas primeras conclusiones tras algo más de dos meses de vida. La corporación pública española da un importante paso adelante en la popularización de un modelo de conexión a Internet llamado a ser común en el futuro. Sin embargo, el HbbTV todavía está lejos de estar completamente implantado y el público parece no conocer todavía todo su potencial.

A pesar de estas circunstancias, lógicas en los albores de una tecnología, se puede concluir que hay una creciente demanda de opciones como el acceso a través de HbbTV a vídeo on demand, lo que muestra que ese servicio puede ser un aliciente central al atraer nuevos públicos y educarlos en el manejo de esta forma de acceso a la red. Además, destaca el intensivo uso infantil del sistema, lo cual constituye una buena noticia, pues los niños suelen ser vectores de tecnificación en los hogares y auténticos impulsores de la renovación tecnológica. Cabe pensar que familiarizándose pronto con las ventajas del HbbTV, contribuirán a su asentamiento definitivo en los próximos años. 
Es también importante tener en cuenta las posibilidades educativas de la televisión conectada: la combinación de emisión lineal de televisión con contenido 'on line' de tipo pedagógico abre la puerta a una nueva generación de herramientas educativas, con un potencial mayor que el que su día tuvieron los CD’s interactivos.

Parece seguro también que los contenidos deportivos son otro de los reclamos para atraer a las audiencias hacia este modelo de televisión conectada. Los grandes eventos deportivos habrán de ser, por tanto, hitos en la expansión del HbbTV en el próximo lustro, y también motivos para la evangelización del público en su uso.

En estos primeros pasos del servicio pionero de RTVE todavía falta por mostrar su potencial la capa de información simultánea interactiva. Esta forma de informarse y de consumir contenidos, simultaneando en la misma pantalla una emisión televisiva con información on line, parece requerir por el momento una curva de aprendizaje mayor que el resto del servicio. Por su novedad, da la impresión de que el público aún no se acostumbra a ella. Sin embargo, es posible que a medida que se combine con otro tipo de contenidos, especialmente de entretenimiento y educativos, su uso cobre mayor sentido y, por tanto, atraiga más interés de la audiencia.

En tanto, aparecen ante los profesionales de la información nuevas y esperanzadoras vías de desempeño laboral en forma de yacimientos de empleo. La concepción y creación de contenidos para este nuevo modelo de consumo exige profesionales mejor formados y estrategias narrativas adaptadas al soporte, por tanto será necesario incorporar a las plantillas estos perfiles laborales.

Por último, resulta evidente la necesidad de abordar nuevas investigaciones sobre el desarrollo del HbbTV y las soluciones que los broadcasters vayan desarrollando. Por las propias condiciones de esta tecnología, un campo que exige mucho trabajo académico es el de la experiencia de usuario: es necesario analizar más en profundidad la relación entre usuario/espectador y los contenidos 'on line' en televisión. La usabilidad, por tanto, requerirá atención en los próximos años. Son necesarios nuevos estudios que identifiquen los patrones de comportamiento del usuario ante esta tecnología, de forma que los desarrollos futuros tengan cimientos más sólidos y permitan construir servicios más eficaces y amplios. Técnicas de medición de la usabilidad, como el eye trackingu otras herramientas heurísticas serán fundamentales para detectar problemas y mejorar en la construcción de futuros servicios como el de RTVE.

Lo mismo cabe decir de la propia unión entre televisión e Internet, que debe dejar de ser una combinación puramente espacial para dar origen a nuevos formatos y géneros. Es necesario que las empresas de comunicación entiendan las posibilidades de este nuevo soporte e inviertan en él. Su capacidad para acortar la brecha digital, llevando Internet a capas de población todavía ajenas a ella, es suficiente argumento como para impulsar el estándar de forma decidida por parte de empresa e instituciones. Además, desde la industria de los contenidos debe entenderse también como un nuevo soporte publicitario y, por tanto, como una nueva forma de monetizar los contenidos y de generar ingresos. 
En esta línea, y habida cuenta de las dificultades que presenta la monitorización de la audiencia en este formato, será necesario investigar formas de contabilizar mejor el tráfico y sumarlo a las mediciones tradicionales de audiencia televisiva, de manera que un programa tenga computado no solo el público que lo vio de forma lineal, sino también el que lo consumió on demand y el que interactuó con él de forma virtual.

Las empresas de contenidos audiovisuales tienen también que entender que este nuevo espacio que se abre en las televisiones requiere de producciones de calidad, bien planificadas en su doble dimensión de productos televisivos y digitales.

En un contexto como el descrito, cabe esperar que narrativas de orden transmedia, televisión social, experiencias de segunda pantalla y otros modelos comunicativos que ahora están siendo apenas ensayados tengan en los próximos años la posibilidad de demostrar su potencial gracias a la proliferación de soportes que ya se vive.

Con todo, la llegada de servicios como el Botón Rojo de RTVE suponen un revulsivo en la ecología de la comunicación, y marcan el camino de la evolución de un medio, la televisión, que parecía ser el último en acomodarse a los tiempos de las nuevas tecnologías y, sin embargo, ha sabido 'aggiornarse' y tomar la delantera a otros. En este sentido, la radio parece llamada a ser el siguiente medio en subirse a la plataforma HbbTV y adaptar sus contenidos a un consumo simultaneado con Internet a través de televisiones conectadas. En esa lógica, radios digitales o híbridas, con conexión a Internet, podrían tener en un futuro inmediato servicios similares.

\section{Referencias bibliográficas}

Álvarez Monzoncillo, J. M. (Coord.) (2011): La televisión etiquetada: nuevas audiencias, nuevos negocios. Barcelona: Ariel.

Alzate, P. (2011): "Hacia una mirada sociocultural del periodismo", Revista Nexus Comunicación, (4). Disponible en: http://dintev.univalle.edu.co/revistasunivalle/index.php/nexus/article/viewFile/1076/1086 [Consultado el 25/10/2013]

Cardoso, G. (2011): Los medios de comunicación en la sociedad en red. Filtros, escaparates y noticias. Barcelona: UOC Ediciones.

Castelló, A. (2013): “El uso de Twitter como canal de información y conversación por parte de los medios convencionales”. Universidad de Alicante. Disponible en: http://www.uchceu.es/actividades_culturales/2013/congresos/documentos/Araceli_Castello_Martinez_1.pdf [Consultado el 02/11/2013].

Castillo, A. M. (2013): “Ficción audiovisual e identidad en las redes sociales”. En Vilches, L. Coord. (2013): Convergencia y transmedialidad. La ficción después de la TDT en Europa e Iberoamérica. Barcelona: Gedisa.

Dufourd, J. C., Thomas S., Concolato, C. (2011): “Recording and delivery of HbbTV applications”. Actas de la 9th International Interactive Conference on Interactive Television. EuroIVT'11 Disponible en: http://tv.tausoft.co.uk/docs/inpro ceedings-2011-11193-3.pdf [Consultado el 20/08/2013]. 
Europa Press (2013): “RTVE lanza ‘Botón rojo', un nuevo servicio para televisiones conectadas a Internet”. Disponible en: http://www.europapress.es/nacional/noticia-rtve-lanza-boton-rojo-nuevo-servicio-televisiones-conectadas-internet20130905164209.html [Consultado el 20/10/2013].

Fondevila Gascón, J. F. (2012): “La televisión conectada: ventajas e inconvenientes”, Cuadernos de Información, n. 31, pp. $11-20$.

Gaitán Moya, J. A, y Piñuel Raigada, J. L. (1998): Técnicas de investigación en comunicación social. Madrid: Síntesis.

García Avilés, J. A. (2012): "Ciudadanos, consumidores, colaboradores y activistas: estrategias de participación de la audiencia en la televisión multiplataforma”. Actas del $3^{\circ}$ Congreso Internacional de la Asociación Española de Investigación de la Comunicación. Disponible en: http://www.aeic2012tarragona.org/comunicacions_cd/ok/205.pdf [Consultado el 15/09/2013].

Harboe, G. (2009): “In Search of Social Television”. En Cesar, P., Geerts, D., y Chorianopoulos, K. (2009): Social interactive television: Immersive shared experiences and perspectives. Hersey: Information Science Reference.

IAB Spain (2013): “Primer estudio anual de Televisión Conectada y vídeo on line”. Disponible en: http:/ /www.iabspain.net/ comision-de-tv-conectada/ [Consultado el 12/11/2013].

Infoperiodistas (2013): “El Botón Rojo de RTVE supera los 600.000 usuarios únicos al mes”. Disponible en: http://www.infoperiodistas.info/noticia/15010/El-boton-rojo-de-TVE-supera-los-600-000-usuarios-unicos-al-mes [Consultado el $12 / 11 / 2013]$

International Telecomunnications Union - ITU (2013): “Integrated broadcast-broadband systems”. Ginebra. Disponible en: http://www.itu.int/dms_pub/itu-r/opb/rep/R-REP-BT.2267-2013-PDF-E.pdf [Consultado el 20/10/2013].

Jonietz, E. (2010): “Making TV Social, virtually”. MIT Technology Review. Disponible en: http:/ www.technologyreview.com/ news/417055/making-tv-social-virtually/ [Consultado el 17/10/2013].

Ludányi, A., Belényesi, P. (2011): “Future trends of television”. Actas de la 22a European Regional ITS Conference. Disponible en: https://www.econstor.eu/dspace/bitstream/10419/52182/1/672616130.pdf [Consultado el 25/08/2013].

Mansilla, V. y Marcos, M. C. (2013): “Experiencia de usuarios en televisión conectada: un estudio con usuarios”, El profesional de la información, v. 22, n. 2, pp. 122-127. Disponible en: http://dx.doi.org/10.3145/epi.2013.mar.04 [Consultado el 26/08/2013].

Medina, M. y Ojer, T. (2011): “La transformación de las televisiones públicas en servicios digitales en la BBC y RTVE”, Revista Comunicar, n. 36, v. XVIII, pp. 87-94.

Merino, A. (2013): “El factor emocional en la narrativa transmedia: la televisión social”, en Fonseca, Journal of Communication, 6. Monográfico, n. 2, pp. 234-257. Disponible en: http://dialnet.unirioja.es/descarga/articulo/4252001.pdf [Consultado el 11/10/2013]. 
Negroponte, N. (2000): El mundo digital. Barcelona: Ediciones B. Panorama audiovisual (2012): "Primer Roland Garros en HbbTV". Disponible en: http://www.panoramaaudiovisual.com/2012/05/18/primer-roland-garros-en-HbbTV/ [Consultado el 20/10/2013].

Prádanos, E. (2011): “La televisión social’: ¿̇tecnología o algo más?”, en Genbeta. Disponible en: http://www.genbeta.com/ redes-sociales-y-comunidades/la-television-social-tecnologia-o-algo-mas-por-eduardo-pradanos [Consultado el $11 / 10 / 2013]$.

Rtve.es (2012): “Histórico despliegue de RTVE.es en Londres 2012 con 19 canales en directo y multiplataforma”. Disponible en: http://www.rtve.es/deportes/20120709/historico-despliegue-rtvees-para-jjoo-londres-19-senales-directo-2200horas-competicion-aplicaciones-para-moviles-tabletas-televisiones/543212.shtml [Consultado el 15/09/2013].

Sánchez Martínez, M. y Barceló Ugarte, T. (2011): “Televisión conectada o de cómo los espectadores se convierten en usuarios: Perspectivas para la interactividad en la industria audiovisual". Actas del $3^{\circ}$ Congreso Internacional Latina de Comunicación Social. Disponible en: http://www.revistalatinacs.org/11SLCS/actas_2011_IIICILCS/021_Barcelo.pdf [Consultado el 18/09/2013].

Scolari, C. (2008): "Hacia la hipertelevisión. Los primeros síntomas de una nueva configuración del dispositivo televisivo", Diálogos de la Comunicación, n. 77. Disponible en: http://dialnet.unirioja.es/descarga/articulo/2694422.pdf [Consultado el 16/10/2013].

Scolari, C. (2013): “La TV después del broadcasting: hipertelevisión, redes y nuevas audiencias”, en Hipermediaciones. Disponible en: http://hipermediaciones.com/2013/06/15/la-tv-despues-del-broadcasting-hipertelevision-redes-y-nuevasaudiencias/ [Consultado el 16/10/2013].

Scolari, C. (2010): "Media Ecology: explorando la metáfora”, en Hipermediaciones. Disponible en: http://hipermediaciones.com/2010/06/24/media-ecology-explorando-la-metafora/ [Consultado el 16/10/2013].

Stefanone, M. A., Lackaff, D. y Rosen, D. (2010): “The Relationship between traditional mass media andsocial media': Reality television as a model for social network site behavior", Journal of Broadcasting y Electronic Media, n. 54, vol. 3, pp. 508-525.

Steffen, H.A. (2012): "Exploring the relationship between engagement and loyalty in the context of social TV”. Tesis de Máster. Universidad de Aalen, Alemania: Disponible en: https://docs.google.com/file/d/0B7GlvcNLfKsJb2JmTE5PNVh1R1U/ edit?pli=1 [Consultado el 20/08/2013].

Toffler, A. (1984): La tercera ola. Barcelona: Plaza y Janés.

Strzebkowski, R., Bartoli, R., y Spiel Vogel, S. (2012): “Demo: connected y social shared smart TV on HbbTV”, en Smart Media Research. Disponible en: http://public.beuth-hochschule.de/ robertst/projekte/Connected-SocialSharedHbbTVapps-Beuth-Paper.pdf [Consultado el 16/10/2013]. 
Vanguardia, La (2013): “TVE presenta la primera aplicación para grabar y compartir en red”. Disponible en: http://www.lavanguardia.com/television/audiencias/20131122/54394400999/tve-aplicacion-nternet-tve.html [Consultado el $22 / 11 / 2013]$.

Vilches, L. (Coord.) (2013): Convergencia y transmedialidad. La ficción después de la TDT en Europa e Iberoamérica. Barcelona: Gedisa. 